\title{
Survey in Novelty Search
}

\author{
Mahmoud Mohamed Nabil \\ Department of computer science \\ Zagazig University \\ Cairo, Egypt
}

\author{
Magdy Zakaria \\ Head of collage of Computers and Information \\ Mansoura University \\ Cairo, Egypt
}

\begin{abstract}
Novelty Search is an algorithm for discovering new things that is motivated by a behavior's novelty. Different generations from the same individual have varying levels of fitness. As a result, the fitness scene is always shifting, and while at the size of a single generation, the euphemism of a fitness landscape with peaks and valleys still remains true, it no longer holds true when seen from the perspective of the entire evolutionary process. What are the characteristics of these algorithms? Is it possible to define a model that will aid in understanding how it functions? This knowledge is necessary for analyzing new Novelty Search versions and current Novelty Search versions, perhaps more effective ones. We claim that in the behaviour space, Novelty Search behaves asymptotically as a uniform random search process. This is an intriguing feature because it's not practical to sample this area directly. The genotype space is only accessible to the algorithm directly, which has a complicated interaction with the behaviour space. On a classic Novelty Search experiment, we discuss the model and put it through its paces. We also show that it puts new light on previous study findings and suggests new research directions.
\end{abstract}

\section{Keywords}

Robotics

\section{INTRODUCTION}

Evolutionary algorithms use a fitness function to guide the selection process, which determines how well an individual meets a set of criteria. This goal-oriented fitness function has been shown to be deceptive $[46,42,41,38]$ and can be substituted having a selection pressure that isn't reliant on the goal. A compulsion to try new things. Amazingly, despite the fact that the goal to achieve is not taken into in any way during the search process. The Novelty Search (NS) procedure that results looks to become at least as effective. $[33,45,53]$. though not as effective than a domain-specific target search such as Swarms of robots, maze navigation and biped locomotion, and plastic neural network design Efficiency scenario changes are a phenomenon that emerges from evolutionary change that are motivated by a particular aim. Individuals tend to reach fitness peaks as a result of the evolutionary process. In Novelty Search, from generation to generation the fitness scene changes. Individuals who were thought to be innovative in one era may not be so in the next. What is an NS process's dynamic? What is the best way to model it? The dearth of solutions to these issues causes serious ramifications. Whenever it comes to putting this an approach into reality. Several important issues remained unsettled such as how to choose behaviour descriptors. What is NS's most important attributes or behaviors? Many practical studies have looked into the impact of various algorithm decisions. However, no conceptual approach has been produced that combines all of these data to provide a holistic perspective of the technique.
It would be fascinating to use such a model to examine the consequences of aesthetic choices, and to suggest more effective methods. In addition, Novelty Search Performance techniques are based on this principle. Rather than an absolutely greatest solution, there are multiple options. These algorithms try to find a diverse group of efficient alternatives. Novelty Search methods and their variants should allow for a thorough investigation of the behaviour space, if not uniform exploration. We present a simplistic and hypothetical paradigm of algorithms that are motivated by novelty. This is based on the notion that Novelty Search this results in an implicit and homogenous stochastic search process in the behaviour space. On the basis of this assumption, a model is constructed, its attributes are studied and explained, and an extension is offered. The importance of these theoretical models is examined in light of the literature after they are compared to experimental results.

\section{BACKGROUND}

\subsection{Swarm robotics}

The study of social insects has inspired the Mobile robots is a subfield of computational intelligence, which is a broader term. Smart technologies for swarms. Whether natural like synthetic insect nest or huge decentralized quadrotors systems depend on its ability to self-organize to demonstrate intelligence conduct that is collectively intelligent as a result, swarm robotics is a promising method for decentralizing the development of cluster automation and the difficulties which have been resolved provide within look at the coordinating of large packs of automated systems. However, designing selforganization in multirobot systems by hand has proven difficult. When manually designing the controlling for single components of a swarming. it is necessary to decompose the macro clustering activity into micro behavioral principles. Discovering significant interactions between individual robots, as well as between robots and the environment, is part of this deconstruction, this will eventually result in the development of universal self-organized conduct. Regrettably, there really is no uniform method for decomposing true international conduct through into clear regulations that control it. As a result, system designers frequently draw Natural swarming mechanisms can be used as motivation, or deliberate guess work can be used [38]. Meta-heuristic methods are applied successfully in several applications such virtual machine placement $[59,60,61,62,63]$.

\subsection{Evolutionary robots}

The use of evolution robots is the application of combinatorial optimization to the creation of mobile robots. Because the need for physical dissection of the intended macro activity is reduced via simulated annealing. Modular robotic platforms can benefit from adaptive robots as a fault diagnosis option. Artificial evolution is simply a process of experimentation in which possible improvements are evaluated assessed based on 
their conduct at the cluster stage. As a result, the adaptive step is followed toward the objective through macro evaluation. Traditional evolutionary techniques, on the other hand, are prone to a variety of flaws. In evolutionary computation, deception is a difficult problem as it can cause the process of evolution to hastily concentrate on unique way. Deception occurs whenever the heuristic generates a false performance slope. Whenever the fitness value lacks to appropriately compensate all stages takes to achieve the optimal solutions, something happens. The bootstrap problem is a potential problem that may arise when using ensemble approach to address challenging situations. Through the early phases of evolution, whenever the job is very onerous to create greater selection pressures on the species. This dilemma arises, due to the fact that everyone performs badly. As a result, there really is no utility slope, and the biological evolution moves at a snail's pace begins to drift towards a boring part of the solution space. gap in an uninspiring part of the solution space Mobility sharing, for example, is a technique for maintaining genomic variety in a population propagation of variety based on the solutions' ranking. Individuals of genetically stages mingling and minimization of genotype age, are one way to avoid deceit. However, the issue could eventually the fitness value will win out over the detection algorithm. Irrespective of how much genomic diversity there is in the population. The process of evolution may nevertheless succeed if the fitness value is intentionally misleading the search. The utilisation of coevolution is a unique technique to dealing with the issue of deceit. Individual fitness is assessed in competitive coevolution instead of an ideal fitness assessment, through rivalry with other members of the population. This in theory, encourages a scramble to the top that drives towards ever solutions. This method has been successful in some sectors, but it is also linked to a variety of concerns arising from potentially counterproductive dynamics among several species that are founder such as poor steady situations divergence. Other approaches for overcoming deception and bootstrapping development rely on breaking down the goal into several sub-goals, each of which is easier to achieve. Incremental evolution, fitness shaping, and multiobjectivization are examples of these strategies. The fact that job division may not always be possible is a common drawback of these methods. Even when it isn't, it requires a large amount of construct significant sub based on past understanding more about work.

\subsection{The Search for Novelty}

Remember that the difficulty with machine learning's objective function is that it doesn't always a recompense for the intermediary starting points which result in the desired destination [37]. Starting points which result in the desired destination. The suggested strategy is to use novelty as a substitute for milestones. That is correct rather than looking for an ultimate goal, the learning technique is awarded for discovering any entity with the ability to differ considerably from what has already been identified. As a result, rather than a novelty metric is used in search utilizing an optimal solution. There is no indicator to quantify increase in this way. In fact, such a method mimics the process of gradual evolution invisibly, namely, progressively amassing fresh forms as they go up the complexity ladder. Initial attempts in a labyrinth navigation domain, for example, may hit a wall and come to a halt. The novelty measure in opposed to a function that is subjective, would credit merely colliding with another barrier, irrespective or not it was better in the future. This type of search necessitates the use of a search engine. a group of instances representing the most recent finds is kept.
Following these characteristic behaviours, further search is launched. After discovering. Although there are a few chosen to implement towards barriers, the only method to get rewarded is to identify a behaviour that does not immediately contact a barrier. From the bottom up, here is how the intricate bucket clogs up. Despite the fact that it is not an objective, a voyager will eventually need to traverse the maze well in order to achieve something new!

This strategy may appear foolish at first look. What assurance is it possible for a search strategy to solve a problem even if the aim isn't specified? Its attractiveness, however, stems from the fact that it rejects the fallacious notion that objectives are a necessary component of the process of discovering. The thought that it's a heavy burden to digest that the aim could be the enemy of progress but if the correct starting points aren't accessible along the route, we'll have little choice but to abandon its delusory illusion of security. What chance is there that innovation will improve things if it offers no information about the solution's path? Isn't the realm of new behaviours limitless, with endless possibilities for meandering? Exhaustive search can be contrasted to novelty search. Naturally, the answer will finally emerge from a search that itemizes all viable solutions. However, it will do so at a huge computational cost. However, there are excellent various grounds to believe that novelty search and thorough search are not the same thing and that the number of innovative acts is sensible and limited in many actual circumstances. The major reason for optimism is that task domains give sufficient limitations on the types of behaviours that can occur or are relevant without the requirement for extra limits imposed by an objective function A maze-navigating robot, for example, can only do so much; the robots in this study only had two effectors. The dimensionality is effectively infinite due to NEAT's ability to absorb additional genes, but the behaviour area into a certain point in the search space implode is restricted. After completing an evaluation in the maze, a robot, for example, comes to a conclusion at a given point. Assume that the robot's behaviour is solely determined by this finishing position. While there are various ways to encode a policy that arrives at a specific point, they all collapse to the same behaviour under this measure of novelty. Indeed, the search space has been condensed to a reasonable number of uniqueness points recognizing the difference between thorough novelty searches. Additionally, rewarding the stepping stones. When overarching goal searching flops, novelty search succeeds. That is to say, everything truly exceptional is praised and encouraged as a launching pad for future growth. If we believe that the basic pathology of overarching goal search is that it is unable to uncover the cornerstones at all, we cannot know which starting points are correct. The pathology is rectified. The novelty search method is introduced in the following section by substituting the optimization model in conjunction with the novelty measurement, as well as the formalization of the notion of novelty.

\subsection{The Novelty Search Algorithm}

Because the population at the heart of evolutionary algorithms like NEAT inherently spans a wide spectrum of growing behaviours $[1,4,6,12,14]$. They're ideal for finding new things. In reality, apart from replacing a uniqueness measurement for the fitness function monitoring novelty involves only minor adjustments to any evolving system. The novelty metric assesses how unique an individual is in comparison to others, putting ongoing pressure on them to try something new. The basic principle is that, rather than rewarding achievement of a 
goal, the novelty search promotes deviation from previous behaviours. As a result, novelty must be assessed.

By examining and quantifying actions to identify their differences, there are numerous potential techniques to measure novelty. Importantly, this measure. It, too, must be domain-specific, much like the fitness function. A new individual's novelty is calculated using the behaviours (rather than the genotypes) of a database of previously generated individuals whose actions were extremely unique at the time of their creation. Furthermore. If you use a stable evolutionary technique. That is the present by replacing one people at a time, individuals can enrich the library with reflecting the far more frequently accessed points. In a novel space, or a space characterized by unusual behaviour. The goal is to determine how distinct the new member seems to be from the general population and predecessors. As a result, any point in the novelty space should be computed by a decent metric. Areas having a higher density the number of segments is deemed fewer distinct, and as a result, they are paid fewer. The average distance to the k-nearest neighbours of a point, where $\mathrm{k}$ is a fixed parameter measured empirically, is a basic measure of sparseness at that point. Intuitively, if the average distance between a particular point's nearest neighbours is big, it is in a sparse area; if the average distance is small, it is in a crowded area. At position $\mathrm{x}$, the sparseness is provided by

$$
f(x)=1 / H \sum_{i=0}^{h} \operatorname{dist}(\mathrm{x}, \text { ? ? } \mathrm{i})
$$

where $\mu \mathrm{i}$ is the ith-nearest neighbor of $\mathrm{x}$ with respect to the distance metric dist, which is a domain-dependent measure of behavioral difference between two individuals in the search space. Individuals from the total population as well as a longterm archive of new members must be considered in the calculation of nearest neighbours [42]. On novelty, individuals from even more scarce portions of the behavioural search space perform better. It's critical to stress that this fresh environment cannot be actively explored. That is, there's no way to know a priori how to access low-density areas, just as there's no way to know a priori how to build a solution that's close to the goal. Exploration is required to navigate the space of novel behaviours. In effect, originality is driven by a coevolutionary dynamic since it is compared to other individuals in the evolutionary process. Similar to archive-based approaches in coevolution, if novelty is sufficiently high at a new person's location, i.e., over a minimal threshold min, the individual is entered into the permanent archive that characterizes the distribution of prior solutions in novelty space. By seeking to the direction of search is basically to what is new in order to maximize the uniqueness index with no other stated objective. It's worth noting that novelty search mimics the common evolutionary computation technique of previous diversity preservation (i.e., speciation). Workout sharing versions are a very well. By lessening selection pressure, these also open up the search. However, unlike Hutter's fitness uniform selection method, these strategies do not take the heretical step of completely ignoring the fitness function. Novelty search, on the other hand, encourages behavioural variation without regard for fitness or an end goal.

It's also worth noting that novelty search isn't a haphazard process; rather, it aims to maximize novelty. Backpedaling is a common occurrence in random walks. Since uniqueness search has an archive that accumulates a trace as to where search has gone, it may be seek to prevent in behaviours spaces of any complexity. In general, the novelty search approach permits any behaviour to be characterized and any novelty measure to be used. Venues with misleading fitness scapes are suitable for novelty search. Intuitive behavioral characterization, and domain limits on feasible expressible actions, notwithstanding its universal applicability. Various search processes will come from altering the way the activity space is specified and comparing characteristics. Comparable to how academics are modifying the goal function to improve search results at the moment.

The point isn't to argue that Practical search is more difficult to set up than surprise search. Rather, The NEAT algorithm resumes regular operation once objective-based fitness is substituted by novelty. Choosing and reproducing the greatest individuals. As the simpler versions are exhausted, the population expands out throughout the range of possible behaviours, ascending to new levels of complexity (i.e., via expanding the neural networks in NEAT) to develop unique behaviours.

\section{CONCLUSIONS}

We found that novelty search often does not produce wellfitting answers, especially whenever the behavioral field contains variables that are unrelated or just distantly related to the aim. We looked into two different forms of novelty searches to solve this problem. Overall, our findings demonstrate that swarm robotics systems can benefit from novelty search in comparison to fitness-driven evolution novelty search has various advantages. One of the most notable benefits is that novelty search frequently results in a wide range of successful self-organizing behaviours. Because developing such behaviours by hand is challenging Variety and self-organization were extremely important in the field of swarming robots. Deception also has no influence on novelty search, is less susceptible to scaling problems, and can build answers with fewer sophisticated neural network models.

\section{REFERENCES}

[1] M. A. Bedau and N. H. Packard. Measurement of evolutionary activity, teleology, and life. In C. G. Langton, C. Taylor, J. D. Farmer, and S. Rasmussen, editors, Proc. of Art. Life II, pages 431-461, Redwood City, CA, 1991. AddisonWesley.

[2] M. A. Bedau, E. Snyder, and N. H. Packard. A classification of longterm evolutionary dynamics. In C. Adami, R. Belew, H. Kitano, and C. Taylor, editors, Pro. of Art. Life VI, pages 228-237, Cambridge, MA, 1998. MIT Press.

[3] Mark Bedau. Four puzzles about life. Artificial Life, 4:125-140, 1998.

[4] A. Channon. Passing the alife test: Activity statistics classify evolution in geb as unbounded. In Proceedings of the European Conference on Artificial Life (ECAL2001). Springer, 2001.

[5] Paul Darwen and Yin Yao. Every niching method has its niche: Fitness sharing and implicit sharing compared. In Hans-Michael Voigt, Werner Ebeling, Ingo Rechenberg, and Hans-Paul Schwefel, editors, Parallel Problem Solving from Nature - PPSN IV, pages 398-407, Berlin, 1996. Springer.

[6] Richard Dawkins. Genetic and evolutionary computation conference (GECCO2007) Keynote Debate, July 2007. 
[7] E. D. De Jong. The incremental pareto-coevolution archive. In Proc. of the Genetic and Evol. Comp. Conf. (GECCO-2004), Berlin, 2004. Springer Verlag.

[8] Jeffrey L. Elman. Incremental learning, or the importance of starting small Technical Report 9101, CRL, La Jolla, CA, 1991.

[9] David E. Goldberg. Simple genetic algorithms and the minimal deceptive problem. In L. D. Davis, editor, Genetic Algorithms and Simulated Annealing, Research Notes in Artificial Intelligence. Morgan Kaufmann, 2007.

[10] Faustino Gomez and Risto Miikkulainen. Incremental evolution of complex general behavior. Adaptive Behavior, 5:317-342, 1997.

[11] Inman Harvey. The Artificial Evolution of Adaptive Behavior. PhD thesis, School of Cognitive and Computing Sciences, U. of Sussex, Sussex, 1993.

[12] Kimberly A. Hughes, Linh Du, F. Helen Rodd, and David N. Reznick. Familiarity leads to female mate preference for novel males in the guppy, poecilia reticulata. Animal Behavior, 58(4):907-916, 1999.

[13] Marcus Hutter and Shane Legg. Fitness uniform optimization. IEEE Transactions on Evolutionary Computation, 10:568-589, 2006.

[14] Michael Lynch. The evolution of genetic networks by non-adaptive processes. Nature Reviews Genetics, 8:803-813, 2007.

[15] Michael Lynch. The frailty of adaptive hypotheses for the origins of organismal complexity. In Proc Natl Acad Sci USA, volume 104, pages 8597-8604, 2007.

[16] C. C. Maley. Four steps toward open-ended evolution. In Proc. of the Genetic and Evol. Comp. Conf. (GECCO1999), pages 1336-1343, San Francisco, 1999.Kaufmann.

[17] Andrew P. Martin. Increasing genomic complexity by gene duplication and the origin of vertebrates. The American Naturalist, 154(2):111-128, 1999.

[18] Daniel W. McShea. Complexity and evolution: What everybody knows. Biology and Philosophy, 6(3):303324, 1991.

[19] Thomas Miconi. Evolution and complexity: The doubleedged sword. Artificial Life: Special Issue on the Evolution of Complexity, 2007.

[20] Thomas Miconi. THE ROAD TO EVERYWHERE: Evolution, Complexity and Progress in Nature and in Computers. PhD thesis, U. of Birmingham, 2007.

[21] Melanie Mitchell, Stephanie Forrest, and John H. Holland. The royal road for genetic algorithms: Fitness landscapes and ga performance. In F. J. Varela and P. Bourgine, editors, Proceedings of the First European Conference on Artificial Life, Cambridge, MA, 1992. MIT Press.

[22] Tom M. Mitchell. Machine Learning. McGraw-Hill, New York, 1997.

[23] C. L. Nehaniv and J. L. Rhodes. On the manner in which biological complexity may grow. In Math. and Comp. Biology, volume 26 of Lectures on Mathematics in the
Life Sciences, pages 93-102. American Mathematical Society, 1999.

[24] T. Ray. Evolution, ecology and optimization of digital organisms. Technical Report Working paper 92-08-042, Santa Fe Institute, 1992.

[25] Russell Standish. Open-ended artificial evolution. International Journal of Computational Intelligence and Applications, 3(167), 2003.

[26] Kenneth O. Stanley, Bobby D. Bryant, and Risto Miikkulainen. Real-time neuroevolution in the NERO video game. IEEE Trans. on Evol. Comp. Special Issue on Evolutionary Computation and Games, 9(6):653-668, 2005.

[27] Kenneth O. Stanley and Risto Miikkulainen. Evolving neural networks through augmenting topologies. Evolutionary Computation, 10:99-127, 2002.

[28] Kenneth O. Stanley and Risto Miikkulainen. A taxonomy for artificial embryogeny. Artificial Life, 9(2):93-130, 2003.

[29] Kenneth O. Stanley and Risto Miikkulainen. Competitive coevolution through evolutionary complexification. Journal of Art. Int. Research, 21:63-100, 2004.

[30] Michiel Van de Panne and Alexis Lamouret. Guided optimization for balanced locomotion. In D. Terzopoulos and D. Thalmann, editors, Sixth Eurographics Workshop on Animation and Simulation, pages 165-177. Springer Verlag, 1995.

[31] James D. Watson, Nancy H. Hopkins, Jeffrey W. Roberts, Joan A. Steitz, and Alan M. Weiner. Molecular Biology of the Gene Fourth Edition. The Benjamin Cummings Publishing Company, Inc., Menlo Park, CA, 1987.

[32] Larry Yaeger. Computational genetics, physiology, metabolism, neural systems, learning, vision and behavior or polyworld: Life in a new context. In C. G. Langton, editor, Art. Life III, Proc. Vol. XVII, pages 263-298. Addison-Wesley,1994.

[33] Stephane Doncieux, Alban Laflaquière, Alexandre Coninx, Novelty Search: A Theoretical Perspective.

[34] Matej Črepinšek, Shih-Hsi Liu, and Marjan Mernik. 2013. Exploration and exploitation in evolutionary algorithms: A survey. ACM Computing Surveys (CSUR) 45, 3 (2013), 35 .

[35] Antoine Cully and Yiannis Demiris. 2018. Quality and diversity optimization: A unifying modular framework. IEEE Transactions on Evolutionary Computation 22, 2 (2018), 245-259.

[36] Agoston E Eiben and Cornelis A Schippers. 1998. On evolutionary exploration and exploitation. Fundamental Informatician 35, 1-4 (1998), 35-50.

[37] Jorge Gomes, Pedro Mariano, and Anders Lyhne Christensen. 2015. Devising effective novelty search algorithms: A comprehensive empirical study. In Proceedings of the 2015 Annual Conference on Genetic and Evolutionary Computation. ACM, 943-950.

[38] Jorge Gomes, Paulo Urbano, and Anders Lyhne Christensen. 2013. Evolution of swarm robotics systems 
with novelty search. Swarm Intelligence 7, 2-3 (2013), $115-144$.

[39] Marc Kirschner and John Gerhart. 1998. Evolvability. Proceedings of the National Academy of Sciences 95, 15 (1998), 8420-8427.

[40] Steijn Kistemaker and Shimon Whiteson. 2011. Critical factors in the performance of novelty search. In Proceedings of the 13th annual conference on Genetic and evolutionary computation. ACM, 965-972.

[41] Joel Lehman and Kenneth O Stanley. 2010. Efficiently evolving programs through the search for novelty. In Proceedings of the 12th annual conference on Genetic and evolutionary computation. ACM, 837-844.

[42] Joel Lehman and Kenneth O Stanley. 2011. Abandoning objectives: Evolution through the search for novelty alone. Evolutionary computation 19, 2 (2011), 189-223.

[43] Joel Lehman and Kenneth O Stanley. 2011. Evolving a diversity of virtual creatures through novelty search and local competition. In Proceedings of the 13th annual conference on Genetic and evolutionary computation. ACM, 211-218.

[44] Joel Lehman and Kenneth O Stanley. 2011. Improving evolvability through novelty search and self-adaptation. In IEEE Congress on Evolutionary Computation. 26932700.

[45] Joel Lehman and Kenneth O Stanley. 2013. Evolvability is inevitable: Increasing evolvability without the pressure to adapt. PloS one 8, 4 (2013), e62186.

[46] Joel Lehman, Bryan Wilder, and Kenneth O Stanley. 2016. On the critical role of divergent selection in evolvability. Frontiers in Robotics and AI 3 (2016), 45.

[47] Antonios Liapis, Georgios N Yannakakis, and Julian Togelius. 2015. Constrained novelty search: A study on game content generation. Evolutionary computation 23, 1 (2015), 101-129. [48] Jean-Baptiste Mouret and Jeff Clune. 2015. Illuminating search spaces by mapping elites. CoRR abs/1504.04909 (2015). arXiv:1504.04909 http://arxiv.org/abs/1504. 04909.

[49] Jean-Baptiste Mouret and Stephane Doncieux. 2012. Encouraging behavioral diversity in evolutionary robotics: An empirical study. Evolutionary computation 20, 1 (2012), 91-133.

[50] Massimo Pigliucci. 2008. Is evolvability evolvable? Nature Reviews Genetics 9, 1 (2008), 75.

[51] Justin K Pugh, Lisa B Soros, and Kenneth O Stanley. 2016. Quality diversity: A new frontier for evolutionary computation. Frontiers in Robotics and AI 3 (2016), 40.

[52] Joseph Reisinger and Risto Miikkulainen. 2007. Acquiring evolvability through adaptive representations. In Proceedings of the 9th annual conference on Genetic and evolutionary computation. ACM, 1045-1052.
[53] Sebastian Risi, Charles E Hughes, and Kenneth O Stanley. 2010. Evolving plastic neural networks with novelty search. Adaptive Behavior 18, 6 (2010), 470491.

[54] Tom Smith, Phil Husbands, and Michael O'Shea. 2003. Local evolvability of statistically neutral GasNet robot controllers. Biosystems 69, 2-3 (2003), 223-243.

[55] Kenneth O Stanley and Risto Miikkulainen. 2002. Evolving neural networks through augmenting topologies. Evolutionary computation 10, 2 (2002), 99127.

[56] Danesh Tarapore, Jeff Clune, Antoine Cully, and JeanBaptiste Mouret. 2016. How do different encodings influence the performance of the MAP-Elites algorithm? In Proceedings of the Genetic and Evolutionary Computation Conference 2016. ACM, 173-180.

[57] Danesh Tarapore and Jean-Baptiste Mouret. 2015. Evolvability signatures of generative encodings: beyond standard performance benchmarks. Information Sciences 313 (2015), 43-61.

[58] Günter P Wagner and Lee Altenberg. 1996. Perspective: complex adaptations and the evolution of evolvability. Evolution 50, 3 (1996), 967-976.

[58] Bryan Wilder and Kenneth Stanley. 2015. Reconciling explanations for the evolution of evolvability. Adaptive Behavior 23, 3 (2015), 171-179. [27] Sewall Wright. 1932. The roles of mutation, inbreeding, crossbreeding, and selection in evolution. In Proceedings of the Sixth International Congress on Genetics. 356-366.

[59] Al-Moalmi, Ammar, Juan Luo, Ahmad Salah, and Kenli Li. "Optimal virtual machine placement based on grey wolf optimization." Electronics 8, no. 3 (2019): 283.

[60] Al-Moalmi, Ammar, Juan Luo, Ahmad Salah, Kenli Li, and Luxiu Yin. "A whale optimization system for energy-efficient container placement in data centers." Expert Systems with Applications 164 (2021): 113719.

[61] Salah A, Shalabi E, Khedr W. A lightweight android malware classifier using novel feature selection methods. Symmetry. 2020 May;12(5):858.

[62] Salah A, Li K, Li K. Lazy-Merge: A Novel Implementation for Indexed Parallel K-Way In-Place Merging. IEEE Transactions on Parallel and Distributed Systems. 2015 Sep 2;27(7):2049-61.

[63] Hosny KM, Salah A, Saleh HI, Sayed M. Fast computation of 2D and 3D Legendre moments using multi-core CPUs and GPU parallel architectures. Journal of Real-Time Image Processing. 2019 Dec;16(6):202741. 